\title{
Étienne Ollion, Raison d'État. Histoire de la lutte contre les sectes en France
}

Jérôme Michalon

\section{Q OpenEdition}

\section{Journals}

\section{Electronic version}

URL: http://journals.openedition.org/lectures/23598

DOI: $10.4000 /$ lectures.23598

ISSN: 2116-5289

\section{Publisher}

Centre Max Weber

Brought to you by Centre national de la recherche scientifique (CNRS)

\section{cnrs}

Electronic reference

Jérôme Michalon, «Étienne Ollion, Raison d'État. Histoire de la lutte contre les sectes en France », Lectures [Online], Reviews, Online since 12 October 2017, connection on 12 November 2020. URL http://journals.openedition.org/lectures/23598; DOI : https://doi.org/10.4000/lectures.23598

This text was automatically generated on 12 November 2020 .

(c) Lectures - Toute reproduction interdite sans autorisation explicite de la rédaction / Any replication is submitted to the authorization of the editors 


\title{
Étienne Ollion, Raison d'État. Histoire de la lutte contre les sectes en France
}

\author{
Jérôme Michalon
}

1 C'est d'un travail de longue haleine qu'Étienne Ollion, chargé de Recherche au CNRS à l'Université de Strasbourg, rend compte dans «Raison d'État ». En effet, l'enquête qui nous est livrée ici, menée dans le cadre d'un mémoire de Master puis d'une thèse de Doctorat, s'est étalée de 2007 à 2012. Et c'est également une histoire longue que cette recherche retrace : celle de la mise en place et de l'évolution, depuis les années 1970, de la lutte contre les groupes qualifiés de sectaires en France. En choisissant un tel objet, É. Ollion a fixé plusieurs objectifs à son travail. Le premier est d'illustrer la fertilité d'une sociologie de l'État (et pas uniquement de l'action publique), et de pointer l'étendue de son pouvoir normatif à travers l'exemple de la lutte contre les sectes et de ses effets sur les groupes en question. Le second objectif, d'ordre disciplinaire, est d'extraire l'objet «sectes» de la sociologie des religions. Pour É. Ollion, réduire la question sectaire à une thématique religieuse est d'une part peu satisfaisant du point de vue empirique, car les politiques françaises en la matière ciblent des comportements précis, dans des domaines variés qui excèdent le seul périmètre de la croyance religieuse, comme certaines formes de psychothérapie. D'autre part, cette réduction des sectes au religieux amène les sociologues des religions à avoir recours à des explications en termes de «culture nationale» pour donner sens au traitement particulier des groupes qualifiés de sectaires en France. Le troisième objectif vise à défendre les vertus de la comparaison internationale pour, précisément, éviter de mobiliser de concepts culturalistes lorsque la sociologie politique peut fournir des descriptions et des outils conceptuels autrement plus convaincants.

2 L'ouvrage se compose de deux grandes parties, suivies d'un épilogue et d'une annexe. La première partie, "Les sectes dans l'État ", est consacrée à la construction de la politique française de lutte contre les groupes sectaires. On y apprend comment, au milieu des années 1970, la première association ADFI (Association de Défense des Familles et des Individus) se crée suite à plusieurs cas de « recrutement » par le groupe Moon, de proches des fondateurs de l'association. On y découvre aussi le peu d'écho que 
suscite l'action de la dite association, du fait tout à la fois de la sociologie de ses membres (situés plutôt à droite et tous catholiques pratiquants), du contexte de l'époque (la vie en "communautés » est un phénomène courant), et du faible nombre d'adhérents aux groupes dénoncés. Le combat contre les sectes apparaît alors comme une lutte morale conservatrice à connotation religieuse, et n'intéresse pas le monde politique. Il faudra que l'association engage tout un travail visant à pathologiser les comportements des personnes recrutées par les groupes sectaires, ces derniers se voyant accusés de «laver le cerveau » de leurs membres. Cette redéfinition nouvelle de la secte, sous l'angle psychologique, permet de laïciser la lutte, et la création d'une seconde association française - le CCMM - Centre d'étude, d'information et d'action contre les Manipulations Mentales- participera de cet élargissement de la cause. Malgré le retentissement du «suicide collectif» d'un millier d'adeptes du People's Temple à Jonestown au Guyana en 1978, il faudra attendre le milieu des années 1990 pour que les groupes sectaires deviennent un problème politique à part entière en France. Une conjoncture particulière a permis cette prise en charge par le monde politique. Le suicide collectif des membres du Temple Solaire en 1995 en France, intervenant quelques mois après l'attaque au gaz sarin par la secte Moon dans le métro de Tokyo, et quelques années après la prise d'otages de Waco au Texas, suscite une forte couverture médiatique. Cet événement coïncide avec le travail d'une mission parlementaire, menée par Jacques Guyard, qui publie quelques mois plus tard un rapport sur la question sectaire. Suivent la reconnaissance d'utilité publique de l'ADFI et la création d'un Observatoire interministériel sur les sectes. En à peine plus d'un an, explique É. Ollion, la thématique sectaire passe du statut d'objet mineur (c'est pour cette raison que J. Guyard s'en était saisi précisément) à celui de problème politique de premier plan, extrêmement consensuel et auquel de plus en plus de parlementaires vont s'intéresser. É. Ollion attire donc l'attention sur l'extrême contingence qui a présidé à la politisation soudaine et spectaculaire de la lutte contre les sectes en France, ce qui affaiblit fortement la pertinence des explications culturalistes de la spécificité française en la matière.

Ollion se démarque encore plus de ces thèses, qui invoquent la supposée tradition laïque ou laïcarde de notre pays pour donner sens à la virulence et à l'ampleur de la réaction politique face aux groupes sectaires. En fait, nous dit l'auteur, les groupes sectaires sont critiqués non parce qu'ils transgressent des normes religieuses, mais surtout parce qu'ils contestent les normes comportementales et intellectuelles dans des domaines pour lesquels l'État assure un rôle de régulation: la santé, l'éducation et les questions de vie en société. La réaction de l'État est d'autant plus forte que les groupes qualifiés de sectaires se sont formés à l'étranger, dans des contextes où le pouvoir normatif de l'État est plus distribué qu'en France. La spécificité française de la lutte contre les sectes s'explique donc, selon ollion, par la forte centralisation du pouvoir public dans l'imposition de normes. Et précisément, on peut mesurer la puissance normative de l'État à l'aune des effets que la politique français en matière de sectes a produit sur les groupes en question. É. Ollion utilise pour cela deux indicateurs: l'évolution du nombre de membres et le nombre d'actes de vandalisme à l'encontre des groupes. Prenant l'exemple des Témoins de Jéhovah, l'auteur décrit une baisse sensible dans le recrutement des membres suite aux publications de rapports parlementaires, de lois, ou d'articles de presse, mais surtout un accroissement spectaculaire des actes de vandalisme. Ce lien très net entre mobilisation politique et infléchissement des 
comportements individuels, montre bien l'étendu du pouvoir symbolique de l'État, dans l'imposition des « bonnes pratiques de soi ».

4 La seconde partie de l'ouvrage, "Une controverse et ses suites", poursuit le récit chronologique de la lutte contre les sectes à partir des années 2000, mais sous un angle différent. Il s'agit ici de retracer la construction d'une controverse précisément à propos de la spécificité de la politique française. À la fin des années 1990, deux organismes internationaux, l'ONU et l'OSCE, font part publiquement de leur inquiétude concernant les libertés religieuses en France. Des groupes qualifiés de sectaires en France, et «inquiétés » à ce titre, sont reconnus comme de "simples » religions dans d'autres pays, en particulier aux États-Unis. Les relations diplomatiques entre les deux pays s'en seraient trouvées un moment ternies. Après enquête, É. Ollion revient sur cette controverse, et nous révèle qu'elle n'a jamais eu lieu; tout du moins pas dans les proportions décrites par les acteurs qui l'ont rapporté en France. C'est à ceux-ci que le sociologue va s'intéresser pour comprendre comment et pourquoi cette «crise diplomatique » a été montée en épingle. Les relations internationales sont donc au centre de cette seconde partie, mais contre toute attente, c'est sur les ONG que l'auteur porte son attention et non plus sur les États. Après avoir expliqué comment le secteur des ONG internationales avait connu un développement important depuis les années 1990, Ollion décrit leur travail de lobbying, mêlant production d'expertise et advocacy. En matière de relations internationales, ces acteurs sont dorénavant indispensables. Certains groupes qualifiés de sectaires, dont les Témoins de Jéhovah et l'Église de Scientologie, vont y avoir recours après avoir expérimenté les tensions suscitées par la politique française et constaté leur incapacité à faire valoir leurs intérêts auprès de la justice du pays. Leur stratégie consiste alors à chercher des soutiens auprès d'organisations États-uniennes, ayant pour leur part réussi à faire adopter l'International Religious Freedom Act en 1998, texte qui s'accompagne de la création d'une commission de surveillance des libertés religieuses à l'échelle mondiale. En 1999, la commission se rendra en France, et ne publiera au final que quelques pages (dans un rapport sur la situation internationale, en comprenant plus de 600). Quelques pages anodines, dont É. Ollion nous dit qu'elles auraient pu rester lettre morte sans la volonté de faire naître une polémique visant à fragiliser la position française en lui donnant artificiellement- des allures de crise diplomatique. Le but de l'opération? Démontrer que la France exerce une politique «discriminatoire» à l'égard de "minorités religieuses ». L'utilisation de ce vocabulaire de la discrimination va de pair avec la stratégie des advocacy officers, travaillant dans les ONG internationales et cherchant à influencer les grandes institutions (ONU, UE, OMS etc.). É. Ollion a suivi l'un d'eux, à Bruxelles, et nous fait découvrir les formes de "mobilisations discrètes " mise en œuvre (présence dans les espaces de rencontre, organisations de colloques, imposition d'éléments de langage etc.) pour faire valoir les intérêts des groupes qualifiés de sectaires. Cette discrétion de l'action fait écho aux évolutions de la politique française dans les années 2000. Les budgets alloués commencent à diminuer à cette époque, et l'unanimité politique face aux sectes s'érode peu à peu. Changement important: la politique ne cible plus les " groupes sectaires » mais les « dérives sectaires ». Il s'agit alors pour l'État de former ses agents (Police, éducation nationale, services sociaux notamment à identifier les comportements suspects, pouvant s'apparenter à ces dérives. Une politique visant moins à rendre public le problème sectaire qu'à produire l' «attention " chez les agents du service public. De la même façon, l'État délègue de plus en plus son action aux associations. É. Ollion parle ainsi d'un mode de 
gouvernement à distance, consistant pour l'État à «faire faire " plutôt qu'à agir directement.

Dans sa conclusion, É. Ollion évoque les évolutions de la lutte contre les sectes en France comme illustratives des capacités d'imposition symboliques de l'État: aujourd'hui, nous dit-il, la question sectaire ne fait plus débat. La dénonciation des comportements sectaires fait largement consensus dans la société française, et c'est le fruit de l'effort politique produit dans les années 1990. En semblant se désinvestir de l'action directe, l'État en réalité prend acte de la diffusion sociale de sa propre manière de problématiser les sectes. É. Ollion souligne l'intérêt d'étudier sur le long terme ce processus en deux phases (politisation et socialisation pourrait-on dire) : l'attention portée uniquement sur la seconde phase débouche sur des explications en termes de «culture nationale ». À cette orientation presque métaphysique, É. Ollion oppose (et propose) une alternative matérialiste : en se penchant sur l'action concrète de l' État, dans la première phase en particulier, il est possible de «rendre compte de la formation de dispositions communes sur le territoire national» (p. 212), voire d'une forme d' « habitus national ».

6 Foisonnant, l'ouvrage d'Étienne Ollion l'est assurément: tant du côté des matériaux d'enquête (archives, statistiques, entretiens, observations) que de la réalité sociale complexe dont il rend compte, sur une période longue de surcroit, l'opus fournit au lecteur une masse d'informations, toujours finement contextualisées, qui viennent assoir la démonstration du sociologue. Convaincant, «Raison d'État » l'est donc aussi, et ce d'autant plus que le propos très clair de l'auteur vient mettre de l'ordre dans le foisonnement des données empiriques. L'argumentation est également très bien menée et montre la fertilité d'une posture d'analyse qui traite des acteurs symétriquement, tout en étant attentive aux asymétries qui existent entre eux, et à l'influence de la critique sociale sur les groupes étudiés. «Raison d'État » est un bel exemple de ce que peut produire la rencontre des acquis de la sociologie dispositionnaliste et des apports de la sociologie pragmatique.

7 Première (petite) ombre au tableau : on n'a absolument aucun mal à adhérer à la thèse concernant la nécessité d'aborder la question sectaire en dehors de la seule sociologie des religions. Les arguments présents dans l'introduction (voire dans la quatrième de couverture) suffisent presque à convaincre le lecteur étranger aux débats entre sociologues des religions et politistes. Si bien que la lecture de l'ouvrage devient en quelques (rares) occasions, fastidieuse. On regrettera également que, malgré une focale annoncée sur l'action de l'État, ce dernier n'apparaisse que comme un acteur parmi d'autres. La majeure partie du terrain effectué nous emmène dans les locaux des associations antisectes, dans les bureaux des lobbyistes, mais finalement assez peu dans les cabinets ministériels. De plus, certains éléments empiriques font défaut pour accréditer l'hypothèse, avancée par l'auteur, d'une inflexion de la politique française (passage des "groupes» aux «comportements ») traduisant le succès de la stratégie polémique décrite plus haut. Enfin, si l'on comprend en quoi les politiques de lutte contre la radicalisation djihadiste constituent, à bien des égards, le prolongement de la lutte contre les sectes, était-il opportun d'y consacrer une partie en forme d'épilogue? Un épilogue sur une actualité "à chaud », qui déroute d'autant plus le lecteur qu'il fait suite à une brillante démonstration sur les vertus analytiques du temps long. 


\section{AUTHOR}

\section{JÉRÔME MICHALON}

Chargé de recherche au CNRS, au laboratoire Triangle (UMR 5206), Jérôme Michalon est sociologue, et travaille sur les rapports humains/animaux, dans une perspective de sociologie des sciences et de sociologie des mobilisations. 